\title{
Hyperspectral Target Detection for Multiple Target Spectra
}

\author{
Edisanter $\mathrm{Lo}^{1+}$ \\ ${ }^{1}$ Department of Mathematical Sciences, Susquehanna University, 514 University Avenue, Selinsgrove, \\ Pennsylvania 17870
}

\begin{abstract}
Target detection is an important application in hyperspectral imaging. Conventional detectors for hyperspectral target detection requires that there is only one target spectrum from a spectral library for detecting a man-made object. The conventional detectors are derived assuming that target and background pixels are from different multivariate normal distributions. This paper proposes a target detection model for detecting a man-made object from multiple target pixels. The model is derived without requiring the assumption of multivariate normal distributions for the pixels. Experimental results using multiple target pixels from a hyperspectral image show that the model can work well in target detection.
\end{abstract}

Keywords: target detection, hyperspectral imaging, remote sensing.

\section{Introduction}

Hyperspectral imaging provides digital images on reflected light. Each pixel in the image is a reflectance spectrum, which can range from several broad spectral bands to several hundreds of narrow spectral bands spanning a specific range in the light spectrum. One of the main applications of hyperspectral imaging in remote sensing is target detection of a known material. Target detection assumes there are only background material and target material in the image, the target material is known, a sample of pixels of background materials can be obtained from the image, and either a sample of pixels or a single pixel of the target material can be obtained from the image or a spectral library. Typically, only a single target pixel is available from a spectral library as a reflectance spectrum.

A review of target detection algorithms is discussed in [1]. The conventional detectors for target detection are the Spectral Angle Mapper (SAM), Adaptive Matched Filter (AMF) [2], and the Adaptive Coherence/Cosine Angle [3]. Some recent detection algorithms are discussed in [4] and [5]. A target detector based on logistic regression is described in [4]. A target detector based on a transformation to a normal distribution is presented in [5]. The SAM detector is defined as the cosine angle between the test pixel and target pixel. The AMF detector assumes common covariance for the background and target distributions, which are assumed to be multivariate normal distributions. The ACE detector is derived from the general likelihood ratio test. The null hypothesis tests if the test pixel has a normal distribution with mean 0 and covariance $C$. The alternative hypothesis tests if the test pixel has a normal distribution with mean as and covariance $\sigma^{2} C$. The vector $s$ is the known target spectrum, and $\sigma^{2}$ and $a$ are unknown scalars.

Conventional detectors, which are derived from the general likelihood ratio test, require multivariate normal distributions for the background and target pixels and also require one target pixel from a target spectral library. Hyperspectral images typically do not have multivariate normal distributions for the background and target pixels. This paper develops a target detector that takes multiple target pixels and does not require normal distributions. The detector is developed in Section 2 and is based on the correlation

+ Corresponding author. Tel.: +1 570-372-4629; fax: +1 570-372-2743.

E-mail address: loe@susqu.edu. 
between the test pixel and target pixels. Experimental results are presented in Section 4 and are obtained by using the detector to detect subpixel targets at different fill factors.

\section{Detection Algorithm}

The target detector based on correlation is presented in this section. Let $y$ be a vector of random variables and denote a test pixel from the image. Let $x_{1}, x_{2}, \ldots, x_{q}$ denote the $q$ target pixels. The image typically consists of mainly background pixels with a few target pixels so that the background mean and covariance can be estimated using the entire pixels from the image. Assume that the test pixel and target pixels have been whitened using a whitening transformation that uses the estimated background mean and covariance. In order to whiten the pixels, the test pixel and each target pixel are assumed to be vectors of random variables. The detector is defined assuming that the test pixel is a realization of a random variable $\boldsymbol{y}$ and the target pixels are realizations of the random variables $x_{1}, x_{2}, \ldots, x_{q}$.

The detector for testing if $\boldsymbol{y}$ is a target pixel or a background pixel using $\boldsymbol{x}_{\boldsymbol{l}}, \boldsymbol{x}_{2}, \ldots, \boldsymbol{x}_{\boldsymbol{q}}$ is defined to be the maximum of the squared correlation between the random variable $y$ and a linear combination of the random variables $\boldsymbol{x}_{1}, \boldsymbol{x}_{2}, \ldots, \boldsymbol{x}_{q}$, i.e.

$$
d(\boldsymbol{x})=\max _{\beta}\left[\operatorname{corr}\left(\boldsymbol{y}, \boldsymbol{x}^{T} \beta\right)\right]^{2}
$$

where $\boldsymbol{x}=\left[\begin{array}{lllll}x_{1} & x_{2} & \ldots & x_{q}{ }^{T}\end{array}\right.$ and $\beta=\left[\begin{array}{lllll}\beta_{1} & \beta_{2} & \ldots & \beta_{q}\end{array}\right]^{T}$ are $q x l$ vectors. The correlation in Equation (1) can be written in terms of covariance as

$$
d(\boldsymbol{x})=\max _{\beta}\left[\frac{\operatorname{cov}\left(\boldsymbol{y}, \boldsymbol{x}^{\boldsymbol{T}} \beta\right)}{\sqrt{\operatorname{cov}(\boldsymbol{y}, \boldsymbol{y})} \sqrt{\operatorname{cov}\left(\beta^{T} \boldsymbol{x}^{T}, \boldsymbol{x}^{T} \beta\right)}}\right]^{2} .
$$

The unknown coefficient $\beta$ is determined by maximizing the squared correlation. The maximum solution for Equation (2) can derived to be the following

$$
d(\boldsymbol{x})=\frac{\operatorname{cov}(\boldsymbol{y}, \boldsymbol{x})[\operatorname{cov}(\boldsymbol{x}, \boldsymbol{x})]^{-1} \operatorname{cov}(\boldsymbol{x}, \boldsymbol{y})}{\operatorname{cov}(\boldsymbol{y}, \boldsymbol{y})}
$$

The detector in Equation (3) has a value between 0 and 1. A large detector value would indicate a potential target.

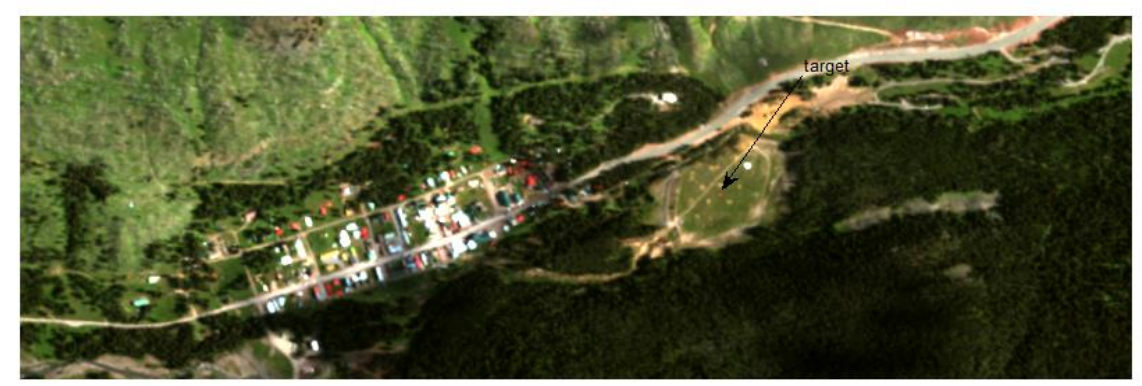

Fig. 1: The RIT image with spatial dimensions of 280 by 800 and spectral dimension of 125 . 
Fig. 2: An image showing the locations of the implanted targets.

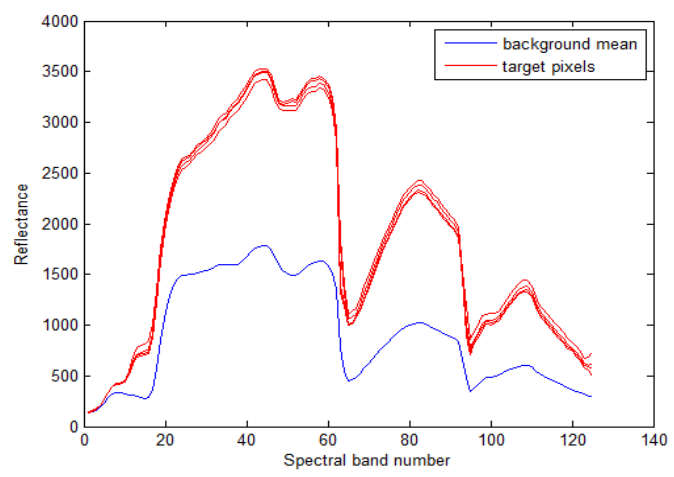

Fig. 3: Plots of the background mean pixel and the 4 target pixels from the image.

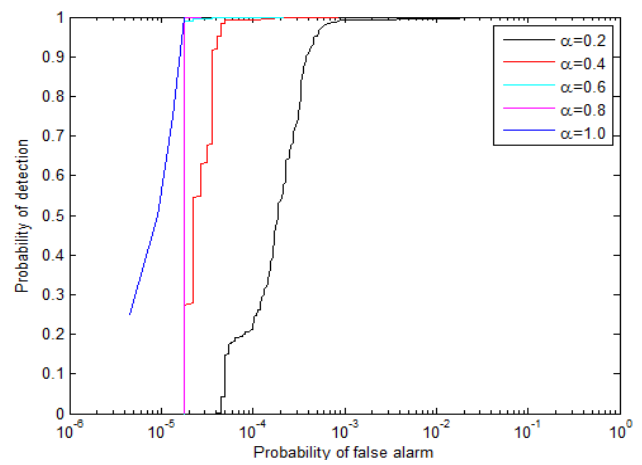

Fig. 4: ROC curves generated by the detector in equation (3) for different values of fill factor $\alpha$.

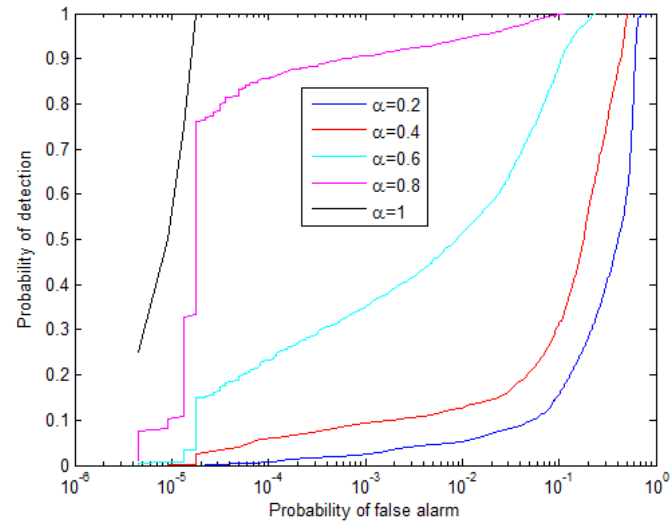

Fig. 5: ROC curves generated by the SAM detector for different values of fill factor $\alpha$. 


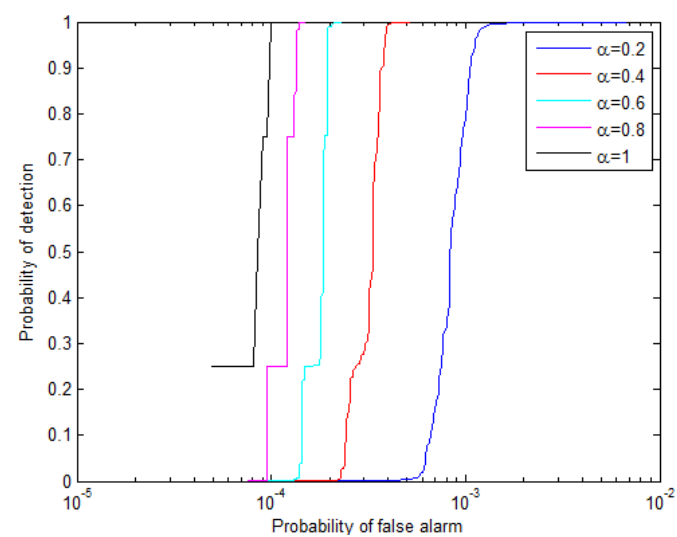

Fig. 6: ROC curves generated by the AMF detector for different values of fill factor $\alpha$.

\section{Experimental Results}

The performance of the detector in Equation (3) on subpixel targets is assessed using a hyperspectral image from RIT (Rochester Institute of Technology) and is compared with the SAM and AMF detectors. ROC curves are generated for the analysis. The image from RIT shown in Figure 1 is the Cooke City image. The arrow points to the four target pixels in the image so $q=4$. The target is a red felt. The image is in the visible and near-infrared wavelengths and has spatial dimensions of 280 by 800 and a spectral dimension of 125. There are not enough test pixels that are subpixel target pixels so 2000 subpixel target pixels are implanted in a random way into the image. The locations of the implanted target pixels are shown in white in Figure 2.

The spectrum of the background mean and the spectra of the four target pixels are shown in Figure 3. The absorption features for both background mean and target spectra are similar. The different fill factors are used for the implanted target pixels. The ROC curves in Figure 4 are generated using the detector in Equation (3) for fill factor $\alpha=0.2,0.4,0.6,0.8,1$. The ROC curves for the SAM and AMF detectors are shown in Figure 5 and Figure 6, respectively, for fill factor $\alpha=0.2,0.4,0.6,0.8$, 1. As the fill factor increases, the performance of all three detectors get better. As more target signal is used in the implanted target pixels, the detectors perform better. The ROC curves show the detector based on correlation perform better than the AMF detector and the AMF detector performs better than the SAM detector. The SAM performs significantly worse than the other two detectors as the fill factor decreases.

\section{Conclusion}

The detector based on maximizing the square of the correlation between the test pixel and multiple target pixels can perform well for test pixels that are subpixel target pixels. The subpixel target pixels are implanted into the image using a user-specified target fill factor. The experimental results using a real hyperspectral image in the visible and near infrared show that the detector performs better than the SAM and AMF detectors.

\section{Acknowledgements}

The author wishes to thank Rochester Institute of Technology for the data.

\section{References}

[1] A. Ziemann and J. Theiler. Simplex ACE: a constrained subspace detector. Optical Engineering 2017, 56(8), 081808

[2] S. Kraut and L. Scharf. The CFAR adaptive sub-space detector is a scale-invariant GLRT. IEEE Trans. Signal Processing 1999, 47: 2538-2541. 
[3] S. Kraut, L.L. Scharf, and L.T. McWhorter. Adaptive subspace detectors. IEEE Trans. Signal Processing 2001, 49: 1-16.

[4] E. Lo and E. Ientilucci. Target detection in hyperspectral imaging using logistic regression. SPIE Proceedings 2016, 9840 (98400W).

[5] E. Lo and E. Ientilucci. Transformation for Target Detection in Hyperspectral Imaging. SPIE Proceedings 2017, $10198(101980 Z)$. 